\title{
A young female with double mechanical prosthetic heart valves presenting with coronary embolism-a rare case report
}

\section{Suman Adhikari'*, Ratna Mani Gajurel', Chandra Mani Paudel', Surya Devkota', Smriti Shakya', Shovit Thapa', Vijay Yadav', Manju Sharma'}

${ }^{1}$ Department of Cardiology, Manmohan Cardiothoracic, Vascular and Transplant Centre, Institute of Medicine, Tribhuwan University, Nepal

Keywords: Acute;Coronary;Embolism;Prosthetic;S yndrome.

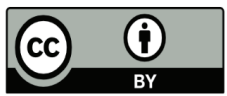

This work is licensed under a Creative Commons Attribution 4.0 Unported License.

\begin{abstract}
Coronary embolism,though uncommon,can occur in young patients with mechanical prosthetic heart valves.Coronary embolism has been reported in patients with prosthetic heart valves with or without thrombosis in literatures.It can cause acute coronary syndrome and lead to death if threshold of diagnosis of coronary embolism is not low. Here we report a case of coronary embolism in a 26 year old female with history of double mechanical prosthetic valve replacement,presenting to our centre with acute coronary syndrome.She was managed with thrombosuction establishing almost complete revascularization.
\end{abstract}

\section{Introduction}

Coronary embolism is a very uncommon cause of acute coronary syndrome and it can have multiple etiologies. The common etiologies include infective endocarditis,atrial fibrillation,prosthetic heart valve thrombosis.There are other less common causes such as iatrogenically induced emboli,atrial myxoma,malignancies. ${ }^{1}$ The incidence of coronary embolism is around $0.06 \% .^{2-3}$ As per literatures,coronary embolism can occur even with normally functioning prosthetic valves when associated with suboptimal anticoagulation. ${ }^{4}$ The left anterior descending artery (LAD) is the most common artery to be involved. ${ }^{1}$ Coronary embolism is responsible for acute coronary syndrome in about 2.9 $\%$ of cases. $^{5}$

\section{Case Report}

A 26 years old female,referred from some remote centre,without any known conventional risk factors for atherosclerotic coronary artery disease presented to us with chief complaints of typical anginal chest pain of 3 days duration. She did not take tablet warfarin for last 3 weeks because of unavailability of the medicines in her place. Her old documents revealed history of double mechanical prosthetic valve replacement at aortic and mitral positions (st. jude bileaflet $32 \mathrm{~mm}$ at mitral and $21 \mathrm{~mm}$ at aortic positions) with tricuspid valve repair three years back for rheumatic heart disease with severe mitral stenosis and moderate aortic regurgitation and moderate tricuspid regurgitation. On examination,patient was anxious due to chest pain. Auscultation revealed preserved metallic clicks. She was hemodynamically stable though. The ECG done in emergency showed ST elevation in I,aVL,V3-V6 and the transthoracic echocardiography revealed hypokinesia of LAD territory with LVEF of $35 \%$ with normally functioning mechanical prosthetic valves (mean pressure gradients across mitral and aortic prosthetic valves were $2.7 \mathrm{mmHg}$ and $13 \mathrm{mmHg}$ respectively) with out any obvious thrombus (we could not perform transesophageal echocardiography though).

Her troponin I level was elevated with value of $1.01 \mathrm{ng} / \mathrm{ml}$. Her INR value was 1.5 (subtherapeutic). The findings were suggestive of acute anterolateral STEMI. As she was having ongoing chest pain, Cath lab was immediately activated and she underwent coronary angiography which surprisingly revealed complete thrombotic occlusion of proximal LAD artery. Then subsequently she underwent thromosuction with plain old balloon angioplasty with establishment of TIMI 3 flow. Patients chest pain gradually subsided and and she was discharged on fifth day of hospitalization

\footnotetext{
${ }^{*}$ Correspondence Author

Dr. Suman Adhikari, MD

ORCID ID NO:0000-0001-8402-5325

Department of cardiology, MCVTC, Institute of Medicine, TUTH, Nepal Phone no. +9779846022044

Email-adhikari11613@gmail.com
} 
with oral anticoagulation.

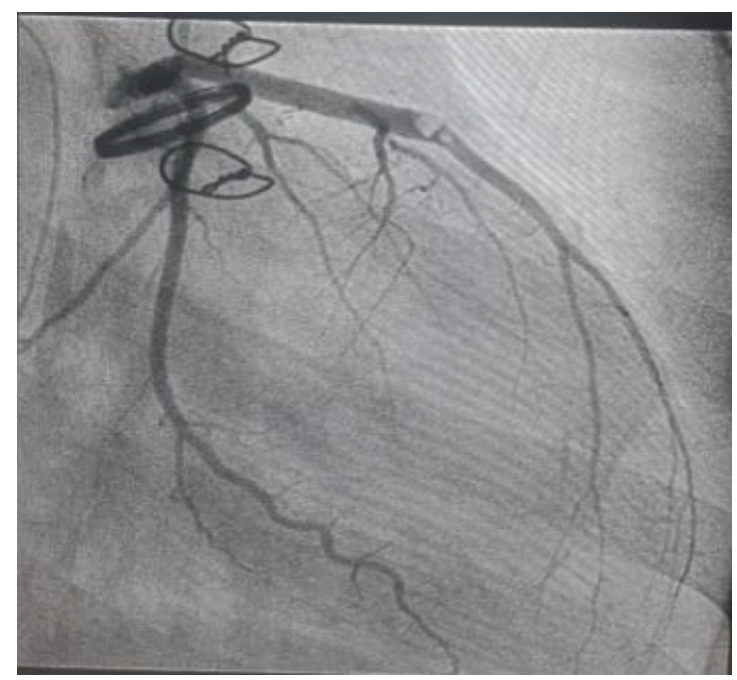

Figure 1-coronary angiography showing a large embolus in proximal LAD.

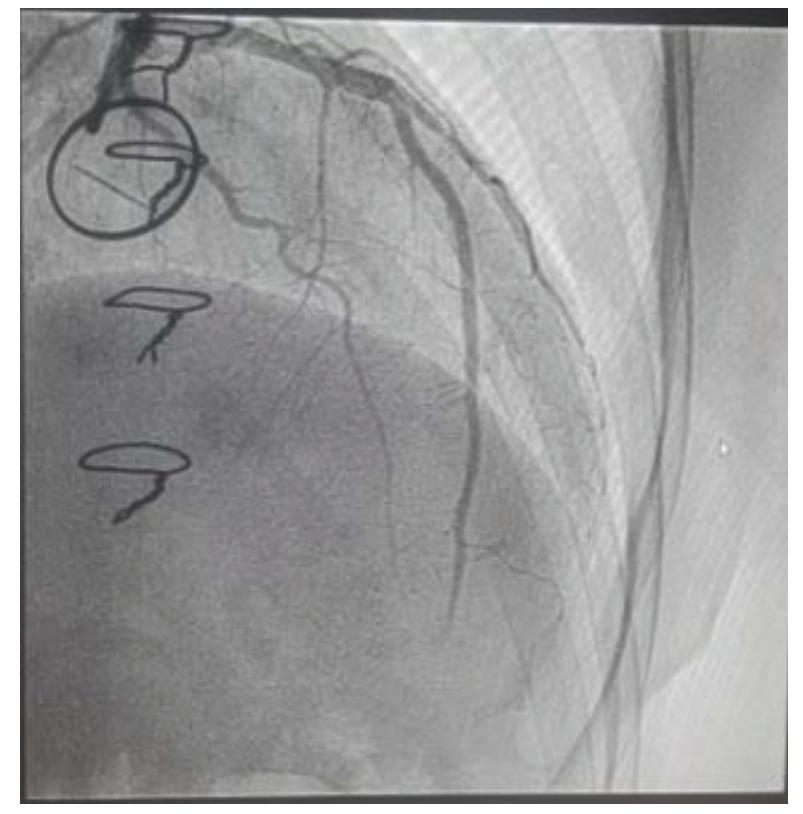

Figure 2-coronary angiography after thrombosuction establishing almost complete revascularization

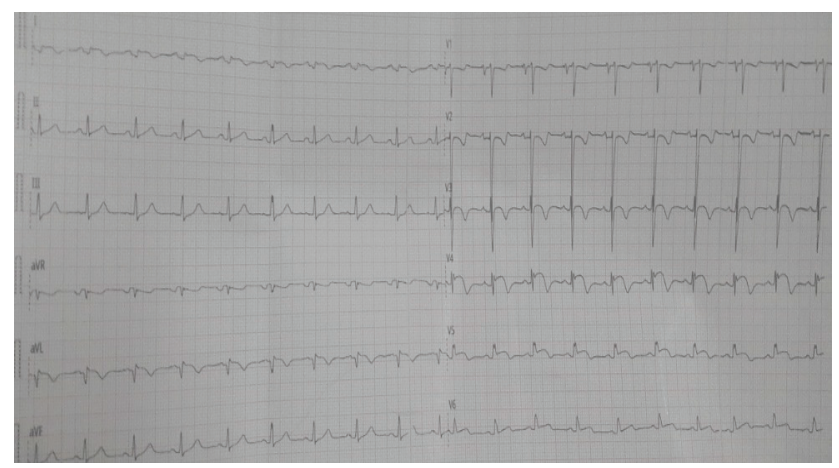

Figure 3-Showing ST segment elevation in leads I,aVL,V3-V6.

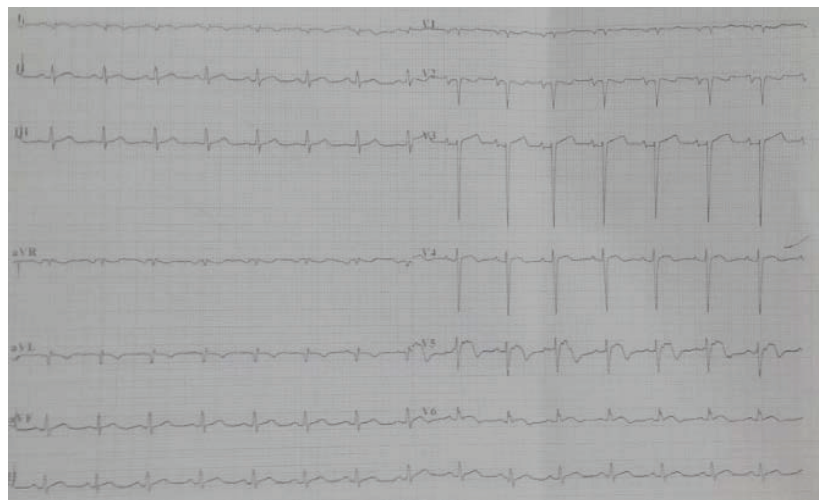

Figure 4-showing resolution of ST segment elevation following revascularization.

\section{Discussion}

Coronary embolism is not reported frequently. There could be various reasons behind it.Important reasons could be high threshold of suspicion for the diagnosis, underappreciation of this phenomenon and underreportings as well. There are very few literatures on acute coronary syndrome with prosthetic heart valves.Many are obtained from case reports/studies. In a case series,only two out of 40 patients with acute coronary syndrome and prosthetic heart valves had normal coronaries with prosthetic heart valve derived coronary emboli. ${ }^{6}$

In autopsies,coronary embolism was found to cause obstruction in $1-17 \%$ cases. ${ }^{7}$ The most common cause of coronary embolism is infective endocarditis. ${ }^{8}$ Emboli due to interventions, myxoma and malignancies are less common causes.'

Coronary embolisation have been reported in patients with prosthetic heart valves dysfunction especially when associated with lack of compliance with oral anticoagulation. ${ }^{4}$ Coronary embolism is more common in left coronary artery system in which LAD is most commonly involved due to its particular course. ${ }^{9}$

The management options, in part, depend upon the etiology of the coronary embolism. The main modalities for management are thrombosuction/aspiration thrombectomy, thrombolysis (not in infective endocarditis), balloon angioplasty with or without stent placement. $^{1}$

\section{Conclusion}

When a patient with history of prosthetic valve placement,especially young patients, with poor compliance to oral anticoagulation(which is more common in developing countries like Nepal)leading to suboptimal anticoagulation present with acute chest pain,one should always lower the threshold for suspecting acute coronary syndrome due to coronary embolism. The early diagnosis in such scenario could save the patients life from fatal outcomes.

\section{Conflict Of Interest:}

\section{None}

Publication History:

Date of submission: April 10, 2020

Date of acceptance: May 23, 2020 


\section{References}

1. Lacey MJ, Raza S, Rehman H, Puri R, Bhatt DL, Kalra A. Coronary Embolism: A Systematic Review. Cardiovascular Revascularization Medicine. 2019 May 22

2. Wenger NK, Bauer S. Coronary embolism: review of the literature and presentation of fifteen cases. The American journal of medicine. 1958 Oct 1;25(4):549-57.

3. Charles RG, Epstein EJ. Diagnosis of Coronary Embolism: A Review1. Journal of the Royal Society of Medicine. 1983 Oct;76(10):863-9.

4. Yazici M, Kayrak M, Turan Y, Koc F, Ulgen MS. Acute coronary embolism without valve thrombosis in a patient with a prosthetic mitral valve--successful percutaneous coronary intervention: a case report. InThe heart surgery forum 2007 (Vol. 10, No. 3, pp. E228-30).

5. Shibata T, Kawakami S, Noguchi T, Tanaka T, Asaumi $Y_{\text {, }}$ Kanaya T, Nagai T, Nakao K, Fujino M, Nagatsuka K, IshibashiUeda H. Prevalence, clinical features, and prognosis of acute myocardial infarction attributable to coronary artery embolism. Circulation. 2015 Jul 28;132(4):241-50

6. lakobishvili Z, Eisen A, Porter A, Cohen N, Abramson E, Mager A, Shapira Y, Sagie A, Battler A, Hasdai D. Acute coronary syndromes in patients with prosthetic heart valves-a caseseries. Acute Cardiac Care. 2008 Jan 1;10(3):148-51

7. Wongrakpanich $S$, Thamcharoen $N$, Chongsathidkiet $P_{\text {, }}$ Siwamogsatham $S$. Dual prosthetic heart valve presented with chest pain: a case report of coronary thromboembolism. Case reports in cardiology. 2015;2015
8. Kraus PA, Lipman J. Coronary embolism causing myocardial infarction. Intensive care medicine. 1990 Mar 1;16(3):215-6.

9. Rathi RK, Bhandari S. Repeated coronary emboli in a young female with mild mitral stenosis in sinus rhythm. International journal of cardiology. 2001 Jul 1;79(2-3):317-9. 